\title{
Utilização de atmosfera modificada na conservação de morangos em pós-colheita ${ }^{(1)}$
}

\author{
Júlio Marques Calegaro(2), Ernani Pezzi( ${ }^{(3)}$ e Renar João Bender(3)
}

\begin{abstract}
Resumo - Foram realizados dois experimentos com o objetivo de testar a utilização de três composições iniciais de atmosferas $\left(3 \mathrm{kPa} \mathrm{O}_{2}+10 \mathrm{kPa} \mathrm{CO}, 5 \mathrm{kPa} \mathrm{O}+15 \mathrm{kPa} \mathrm{CO}_{2}\right.$ e do ar ambiente) com dois tempos de armazenagem (7 e 14 dias) a $0^{\circ} \mathrm{C}$, para a conservação de morangos (Fragaria x ananassa Duch.) cv. Oso Grande. Os frutos eram provenientes de uma lavoura comercial do Município de Farroupilha, RS. No primeiro experimento, utilizaram-se vidros de $1.700 \mathrm{~mL}$ de capacidade para a armazenagem, e no segundo, empregaram-se sacos de polietileno, de $53 \mathrm{~cm}$ x $90 \mathrm{~cm}$ e $90 \mu \mathrm{m}$ de espessura. As atmosferas com $3 \mathrm{kPa} \mathrm{O}_{2}+10 \mathrm{kPa} \mathrm{CO}$ ou $5 \mathrm{kPa} \mathrm{O}_{2}+15 \mathrm{kPa} \mathrm{CO}_{2}$ apresentaram bom potencial para a conservação dos frutos por até 7 dias. A modificação da atmosfera em embalagens de polietileno empregando concentrações iniciais de ar ambiente aumentou o período de conservação e manteve a qualidade dos frutos por até 7 dias. Os benefícios decorrentes da utilização dessas atmosferas foram a manutenção da firmeza da polpa, da coloração dos frutos, dos teores de açúcares totais, e de ácido ascórbico.
\end{abstract}

Termos para indexação: Fragaria x ananassa, frutas, armazenamento em atmosfera controlada, refrigeração, qualidade do produto.

\section{Use of modified atmosphere storage on postharvest quality of strawberries}

\begin{abstract}
Two experiments were conducted to test the use of three initial compositions of storage atmospheres $\left(3 \mathrm{kPaO}_{2}+10 \mathrm{kPa} \mathrm{CO}_{2}, 5 \mathrm{kPa} \mathrm{O}+15 \mathrm{kPa} \mathrm{CO}_{2}\right.$ or ambient air) and two storage periods (7 or 14 days) at $0^{\circ} \mathrm{C}$ on the quality of strawberries (Fragaria $\mathrm{x}$ ananassa Duch.) cv. Oso Grande. The fruits were originated from a grove of Farroupilha, RS, Brazil. In the first experiment, strawberries were stored in glass bottles with $1,700 \mathrm{~mL}$ of capacity. In the second experiment, polyethylene bags of $53 \mathrm{~cm} \times 90 \mathrm{~cm}$ and $90 \mu \mathrm{m}$ of thickness were used. The use of atmospheres with $3 \mathrm{kPa} \mathrm{O}_{2}+10 \mathrm{kPa} \mathrm{CO}_{2}$ or $5 \mathrm{kPa} \mathrm{O}_{2}+15 \mathrm{kPa} \mathrm{CO}_{2}$ resulted in a good storage potential of strawberries for up to 7 days. The modification of the atmosphere in polyethylene bags, using initial concentrations of ambient air also resulted in adequate fruit quality up to 7 days. The use of these atmospheres resulted in the maintenance of the pulp firmness, good fruit color and higher contents of total sugars and of ascorbic acid.
\end{abstract}

Index terms: Fragaria $\mathrm{x}$ ananassa, fruits, controlled atmosphere storage, refrigeration, product quality.

\section{Introdução}

A rápida deterioração pós-colheita de morangos em temperatura ambiente tem sido atribuída à eleva-

\footnotetext{
(1) Aceito para publicação em 5 de novembro de 2001.

Extraído da dissertação de mestrado apresentada pelo primeiro autor à Universidade Federal do Rio Grande do Sul (UFRGS), Porto Alegre, RS. Financiado pela Fapergs.

(2)UFRGS, Fac. de Agronomia, Caixa Postal 776, CEP 91510-970 Porto Alegre, RS. Bolsista CNPq. E-mail: jmcalegaro@bol.com.br

(3) UFRGS, Fac. de Agronomia, Dep. de Horticultura e Silvicultura, Caixa Postal 776, CEP 91510-970 Porto Alegre, RS. E-mail: stern@cpovo.net, rjbe@vortex.ufrgs.br
}

da taxa respiratória e ao aumento da produção de etileno (Kader, 1992). Outros fatores, como a suscetibilidade à lesão mecânica, a perda de água (Nunes et al., 1995) e a deterioração causada por fungos, especialmente Botrytis cinerea (El-Kazzaz et al., 1983), contribuem para diminuir o período de conservação. Além desses fatores, alterações na cor, na firmeza da polpa e perda do brilho natural também são observadas após a colheita. Por ser um produto de alto valor comercial, principalmente no mercado in natura, o morango requer a utilização de tecnologia adequada para melhor conservação. A utilização de baixas temperaturas pode auxiliar no aumento do tempo de conservação pós-colheita; 
porém, em temperaturas apropriadas de 0 a $1^{\circ} \mathrm{C} \mathrm{e}$ umidade relativa do ar (UR) de 90 a $95 \%$, a vida de armazenagem do morango é de apenas 7 dias (Nunes et al., 1995). Contudo, o emprego de baixas temperaturas serve também como base para métodos complementares de conservação dos frutos, tais como o controle ou a modificação da atmosfera, a irradiação e os tratamentos químicos, que, se utilizados isoladamente, muitas vezes não surtem efeito satisfatório (Botrel, 1994). O emprego de atmosferas com concentrações de 5-10\% de $\mathrm{O}_{2}$ e de $15-20 \%$ de $\mathrm{CO}_{2}$ tem sido recomendado porque pode aumentar o tempo de conservação dos morangos, mantendo a sua qualidade (Kader, 1992). Os benefícios para a conservação dos frutos incluem a redução da taxa respiratória (Li \& Kader, 1989) e da produção de etileno (Kim \& Wills, 1998), além do retardamento da perda de cor e firmeza dos frutos (Li \& Kader, 1989; Smith, 1992; Smith \& Skog, 1992; Larsen \& Watkins, 1995) e do crescimento fúngico (El-Kazzaz et al., 1983). Entretanto, tem-se observado que exposições prolongadas dos morangos a atmosferas com altos níveis de $\mathrm{CO}_{2}$ ou baixos de $\mathrm{O}_{2}$ podem levar ao surgimento de sabores e odores indesejados e à perda de coloração dos frutos (Li \& Kader, 1989; Ke et al., 1993). Desse modo, a busca de métodos que proporcionem maior tempo de conservação, mantendo as boas características de qualidade e aparência dos frutos, são fundamentais.

O objetivo deste trabalho foi avaliar o uso de diferentes composições iniciais de atmosferas e tempos de armazenagem refrigerada para conservação de morangos cultivar Oso Grande, após a colheita.

\section{Material e Métodos}

Foram realizados dois experimentos com morangos da cv. Oso Grande, provenientes de uma lavoura comercial localizada no Município de Farroupilha, RS. O delineamento experimental utilizado nos dois experimentos foi o completamente casualizado, em esquema fatorial $3 \times 2$ (três composições de atmosferas e dois tempos de armazenagem), com quatro repetições.

No experimento 1, os morangos foram colhidos no dia 16 de fevereiro de 2000 e imediatamente transportados, em veículo sem refrigeração, para o Laboratório de Pós-Colheita do Departamento de Horticultura e Silvicultura da Faculdade de Agronomia da UFRGS, em Porto Alegre, distante $120 \mathrm{~km}$ do local de colheita.
No laboratório, os morangos sadios e sem danos foram distribuídos em três conjuntos de oito vidros de $1.700 \mathrm{~mL}$ de capacidade, procurando-se uniformizar o número de frutos (30-40 frutos) nesses recipientes. Os vidros de dois desses conjuntos foram fechados, lacrados com parafilme e ligados em série por mangueiras a cilindros contendo misturas de $3 \mathrm{kPaO}_{2}+10 \mathrm{kPaCO}$ ou $5 \mathrm{kPaO}_{2}+$ $15 \mathrm{kPa} \mathrm{CO}_{2}$. O tempo de passagem dos gases foi de uma hora. No terceiro conjunto, os vidros permaneceram sem tampa, em presença do ar ambiente (controle). Em seguida, todos os vidros foram colocados em câmara B.O.D com a temperatura ajustada para $1^{\circ} \mathrm{C}$, e quatro vidros de cada atmosfera experimental ficaram armazenados por 7 dias, e os restantes, permaneceram por 14 dias.

No experimento 2, os morangos foram colhidos no dia 23 de janeiro de 2001, colocados em bandejas de plástico, cobertos com película de PVC, e armazenados em câmara frigorífica $\left(0^{\circ} \mathrm{C}\right)$, na mesma propriedade. No dia seguinte, foram transportados, em veículo sem refrigeração, até o Instituto de Ciência e Tecnologia de Alimentos da UFRGS, em Porto Alegre, RS, distante $120 \mathrm{~km}$ do local de produção. Os tratamentos constituíram-se da combinação de misturas de gases $\left[3 \mathrm{kPaO}_{2}+10 \mathrm{kPaCO}_{2}, 5 \mathrm{kPa} \mathrm{O}+\right.$ $15 \mathrm{kPa} \mathrm{CO}_{2}$ e ar ambiente (controle)] e tempos de armazenagem refrigerada (7 e 14 dias), com quatro repetições. A unidade experimental consistiu de uma bandeja com capacidade para $400 \mathrm{~g}$ (35-40 frutos). As bandejas (sem a película de PVC) de cada tratamento foram acondicionadas em caixas de papelão $(44 \times 33 \times 10 \mathrm{~cm})$ e envolvidas em um saco de polietileno de $53 \mathrm{~cm}$ x $90 \mathrm{~cm}$ e $90 \mu \mathrm{m}$ de espessura. $\mathrm{O}$ ar das embalagens foi retirado com bomba de vácuo, sendo, a seguir, injetada a mistura de $3 \mathrm{kPa} \mathrm{O}_{2}+$ $10 \mathrm{kPa} \mathrm{CO}_{2}$ ou a mistura de $5 \mathrm{kPa} \mathrm{O}_{2}+15 \mathrm{kPa} \mathrm{CO}_{2}$, provenientes de cilindros de $9 \mathrm{~m}^{3}$, que foram logo fechados. No tratamento-controle, as embalagens foram apenas insufladas com ar e, em seguida, lacradas e armazenadas em câmara frigorífica, a $0^{\circ} \mathrm{C}$, por 14 dias.

As variáveis estudadas foram a perda de peso, a intensidade de deformação de polpa, a coloração da epiderme, a acidez total titulável (ATT) e os teores de açúcares totais e ácido ascórbico (vitamina C). A intensidade de deformação da polpa foi determinada pelo medidor de deformação da polpa de Hamson (1952), em 21 morangos de cada repetição. A determinação da coloração da epiderme dos frutos [intensidade da cor (croma) e ângulo da intensidade da cor (h)] foi feita apenas no experimento 2, em uma única bandeja de cada tratamento, após cada período de armazenagem, utilizando-se um colorímetro (modelo CR 300, Minolta). 
$\mathrm{Na}$ determinação da ATT, subamostras de $6,0 \mathrm{~g}$ do homogeneizado do tecido de cada repetição foram diluídas em $100 \mathrm{~mL}$ de água destilada. A ATT, expressa em porcentagem de ácido cítrico, foi determinada pela titulação com solução de $\mathrm{NaOH} 0,1 \mathrm{~N}$ até pH 8,1. Os teores de açúcares totais foram determinados pelo método Fenol-Ácido Sulfúrico (Dubois et al., 1956), com leitura da absorbância a $490 \mathrm{~nm}$ em espectrofotômetro. Os teores de ácido ascórbico (total) foram determinados pelo método de 2,4-dinitrofenilidrazina (Tereda et al., 1978), com leitura da absorbância a $540 \mathrm{~nm}$ em espectrofotômetro. Os dados de cada variável foram submetidos à análise de variância, e as médias, comparadas estatisticamente pelo teste de Duncan a $5 \%$ de probabilidade, utilizando o programa SANEST (Sistema de Análise Estatística) da Esalq/USP. A média da amostragem inicial, obtida por ocasião da instalação do experimento, foi comparada com a média geral, tanto dos tratamentos armazenados por 7 dias como dos armazenados por 14 dias, pelo teste t.

\section{Resultados e Discussão}

Na avaliação da variável perda de peso dos tratamentos do experimento 1 , houve interação significativa entre composição da atmosfera e tempo de armazenagem (Tabela 1). Os frutos mantidos em ar

Tabela 1. Perda de peso (\%) de morangos cv. Oso Grande mantidos em diferentes composições iniciais de atmosferas e tempos de armazenagem a $1^{\circ} \mathrm{C}$ (Experimento 1). Médias de quatro repetições. Porto Alegre, 2000 ${ }^{(1)}$.

\begin{tabular}{|c|c|c|}
\hline \multirow[t]{2}{*}{ Composição da atmosfera } & \multicolumn{2}{|c|}{ Tempo de armazenagem (dias) } \\
\hline & 7 & 14 \\
\hline Ar ambiente & $17,1 \mathrm{bA}$ & $23,7 \mathrm{aA}$ \\
\hline $3 \mathrm{kPa} \mathrm{O}_{2}+10 \mathrm{kPa} \mathrm{CO}$ & $2,4 \mathrm{aB}$ & $2,8 \mathrm{aB}$ \\
\hline $5 \mathrm{kPa} \mathrm{O}_{2}+15 \mathrm{kPa} \mathrm{CO}_{2}$ & $1,8 \mathrm{aB}$ & $1,8 \mathrm{aB}$ \\
\hline
\end{tabular}

(1)Médias seguidas da mesma letra, minúscula na linha e maiúscula na coluna, não diferem entre si pelo teste de Duncan a $5 \%$ de probabilidade. ambiente apresentaram maior perda de peso em relação aos das outras duas atmosferas experimentais, tanto aos 7 quanto aos 14 dias de armazenagem. Diferenças significativas dentro da mesma composição da atmosfera só foram observadas entre os tratamentos mantidos em ar ambiente, e as maiores perdas se deram aos 14 dias. Robinson et al. (1975), citado por García et al. (1998), relataram que a máxima perda de peso comercialmente tolerada para morangos é de 6\%. De acordo com esse critério, constatase que tanto os frutos mantidos nas atmosferas iniciais de $3 \mathrm{kPa} \mathrm{O}_{2}+10 \mathrm{kPa} \mathrm{CO}_{2}$, como de $5 \mathrm{kPa} \mathrm{O}_{2}+$ $15 \mathrm{kPa} \mathrm{CO}_{2}$, apresentaram valores abaixo do tolerado.

No experimento 2, diferenças significativas de perda de peso foram constatadas apenas entre tempos de armazenagem. Os tratamentos armazenados por 14 e 7 dias apresentaram uma perda de peso média de $2,3 \%$ e $0,9 \%$, respectivamente. Nenhum tratamento apresentou perda de peso acima do valor tolerado em morangos, segundo Robinson et al. (1975), citados por García et al. (1998).

Quanto à intensidade de deformação de polpa no experimento 1 , constatou-se que os frutos mantidos em ar ambiente apresentaram maior deformação da polpa, em relação aos frutos das outras duas atmosferas experimentais (Tabela 2). É possível que a maior intensidade de deformação de polpa dos morangos mantidos em ar ambiente tenha decorrido da diminuição da turgescência dos tecidos, como conseqüência da elevada transpiração dos frutos. No experimento 2, houve interação significativa entre composição da atmosfera e tempo de armazenagem em relação à intensidade de deformação de polpa (Tabela 3). Aos 7 dias de armazenagem, não hou-

Tabela 2. Intensidade de deformação de polpa (IDP), acidez total titulável (ATT), teores de açúcares totais (AT) e de ácido ascórbico (AA) de morangos cv. Oso Grande armazenados em diferentes composições iniciais de atmosferas e tempos de armazenagem a $1^{\circ} \mathrm{C}$ (Experimento 1). Porto Alegre, 2000 ${ }^{(1)}$.

\begin{tabular}{|c|c|c|c|c|c|}
\hline \multirow[t]{2}{*}{ Variável } & \multicolumn{3}{|c|}{ Composição da atmosfera } & \multicolumn{2}{|c|}{ Tempo de armazenagem (dias) } \\
\hline & Ar ambiente & $3 \mathrm{kPa} \mathrm{O}_{2}+10 \mathrm{kPaCO}$ & $5 \mathrm{kPa} \mathrm{O}_{2}+15 \mathrm{kPaCO}_{2}$ & 7 & 14 \\
\hline IDP (mm) & $0,51 \mathrm{a}$ & $0,11 \mathrm{~b}$ & $0,10 \mathrm{~b}$ & - & - \\
\hline ATT (\% de ácido cítrico) & $0,92 \mathrm{a}$ & $0,66 \mathrm{~b}$ & $0,67 \mathrm{~b}$ & - & - \\
\hline AT (mg/g de amostra) & $55,9 \mathrm{a}$ & $37,8 b$ & $37,7 b$ & $48,6 \mathrm{a}$ & $39,0 \mathrm{~b}$ \\
\hline AA (mg/100 g de amostra) & $59,4 \mathrm{a}$ & $46,4 \mathrm{~b}$ & $44,1 \mathrm{~b}$ & $53,3 \mathrm{a}$ & $46,7 \mathrm{~b}$ \\
\hline
\end{tabular}

${ }^{(1)}$ Médias seguidas da mesma letra não diferem entre si pelo teste de Duncan a $5 \%$ de probabilidade. 
ve diferenças entre as atmosferas experimentais. Entretanto, aos 14 dias os frutos do tratamento-controle apresentaram-se mais suscetíveis à deformação do que os das outras duas atmosferas, as quais não diferiram entre si. Larsen \& Watkins (1995) também constataram que morangos cv. Pajaro, armazenados a $0^{\circ} \mathrm{C}$ em atmosfera com $20 \%$ de $\mathrm{CO}_{2}$, estavam mais firmes, após 12 dias, do que os frutos armazenados em ar ambiente. Os resultados do presente estudo indicam uma possível contribuição das atmosferas com concentrações iniciais de $3 \mathrm{kPa} \mathrm{O}_{2}+10 \mathrm{kPa} \mathrm{CO}$ e $5 \mathrm{kPa} \mathrm{O}_{2}+15 \mathrm{kPa} \mathrm{CO}$ na manutenção da firmeza, já que não houve diferença significativa na intensidade de deformação da polpa com o aumento do tempo de armazenagem. A manutenção da firmeza da polpa dos morangos é um importante atributo de qualidade no manejo pós-colheita. Frutos mais firmes, em geral, estão associados a uma melhor conservação e aspecto visual, sendo, portanto, preferidos pelos consumidores.

$\mathrm{Na}$ avaliação da cor da epiderme dos morangos do experimento 2 , nenhuma diferença significativa foi encontrada entre os tratamentos e entre a amostra inicial e os tratamentos. Os valores médios do "croma" (pureza da cor) e "h" (intensidade da cor) da amostra inicial foram de 41,2 e $33,2^{\circ}$, respectivamente, enquanto os valores de "croma" e "h" da média geral dos tratamentos foram de 43,2 e 33, $6^{\circ}$. Os valores de pureza semelhantes mostraram que não houve escurecimento acentuado dos frutos, enquanto os valores de intensidade da cor semelhantes indicaram que a cor vermelha dos frutos manteve-se praticamente a mesma em relação ao início do experimento. Nunes \& Morais (1995), García et al. (1998) e

Tabela 3. Intensidade de deformação de polpa de morangos cv. Oso Grande mantidos em diferentes composições iniciais de atmosfera e tempos de armazenagem a $0^{\circ} \mathrm{C}(\mathrm{Ex}-$ perimento 2). Valores expressos em milímetros de deformação de polpa. Médias de quatro repetições. Porto Alegre, $2001^{(1)}$.

\begin{tabular}{lcc}
\hline Composição da atmosfera & \multicolumn{2}{c}{ Tempo de armazenagem (dias) } \\
\cline { 2 - 3 } & \multicolumn{1}{c}{7} & 14 \\
\hline Ar ambiente & $0,40 \mathrm{bA}$ & $0,88 \mathrm{aA}$ \\
$3 \mathrm{kPa} \mathrm{O}+10 \mathrm{kPa} \mathrm{CO}_{2}$ & $0,49 \mathrm{aA}$ & $0,51 \mathrm{aB}$ \\
$5 \mathrm{kPa} \mathrm{O}_{2}+15 \mathrm{kPa} \mathrm{CO}_{2}$ & $0,48 \mathrm{aA}$ & $0,61 \mathrm{aB}$ \\
\hline
\end{tabular}

${ }^{1)}$ Médias seguidas da mesma letra, minúscula na linha e maiúscula na coluna, não diferem entre si pelo teste de Duncan a 5\% de probabilidade.
Sanz et al. (1999) também não observaram alteração tanto na cor como no escurecimento dos morangos, armazenados por sete, quatro e sete dias, respectivamente. A manutenção da cor dos morangos durante o armazenamento é outro atributo de qualidade desejado, já que o escurecimento excessivo dos frutos compromete seu aspecto visual e, portanto, a sua aceitação pelo consumidor.

Em relação à ATT, diferenças significativas foram encontradas apenas entre composições de atmosferas no experimento 1 (Tabela 2). Os maiores valores de ATT encontrados nos tratamentos com ar ambiente devem estar relacionados à maior perda de água pelos frutos, resultando na concentração dos ácidos orgânicos presentes no suco celular. Na comparação da amostragem inicial com os tratamentos armazenados, tanto aos 7 dias como aos 14 dias, não foram encontradas alterações significativas de ATT. No experimento 2, não houve diferenças significativas na ATT entre os tratamentos, pois o valor médio encontrado nessa variável foi de $0,89 \%$. Na comparação da amostra inicial com os tratamentos armazenados por 7 e 14 dias, foi observado um aumento na ATT com o aumento do tempo de armazenagem. Entretanto, diferenças significativas só foram constatadas entre a amostra inicial $(0,84 \%)$ e os tratamentos armazenados por 14 dias $(0,92 \%)$; o valor médio encontrado nos tratamentos armazenados por 7 dias foi de $0,87 \%$. O aumento da ATT dos tratamentos armazenados por 14 dias deve estar relacionado com a perda de água dos frutos. Weichman (1986), citado por Nunes \& Morais (1995), mencionou que os ácidos orgânicos tendem a diminuir e não a aumentar, durante o amadurecimento dos frutos, em virtude da utilização como substrato para a respiração. Desse modo, o aparente aumento da ATT observado aos 14 dias deve ser conseqüência não da síntese de ácidos orgânicos, mas sim do efeito da concentração, em razão da perda de água pelos frutos. Embora o efeito de concentração mencionado anteriormente deva ser considerado, é possível que os teores de ácidos orgânicos não tenham diminuído significativamente ao longo do tempo de armazenagem. Sendo assim, os resultados do presente experimento foram indicativos de que as concentrações de gases no interior das embalagens podem ter contribuído para retardar a atividade respiratória, 
reduzindo, assim, o consumo de reservas de ácidos orgânicos. Esse efeito pode ter ocorrido mesmo entre os tratamentos com ar ambiente, cujas concentrações iniciais de $\mathrm{O}_{2}$ e $\mathrm{CO}_{2}$ alteram-se com a atividade respiratória dos frutos, enquanto armazenados, atingindo níveis que acabaram influenciando o metabolismo.

Na avaliação da variável ácido ascórbico, no experimento 1, diferenças significativas foram encontradas, tanto entre tempos de armazenagem como entre composições da atmosfera (Tabela 2). Teores mais elevados desse ácido, encontrados nos tratamentos mantidos em ar ambiente, podem ser atribuídos à grande perda de água verificada nos frutos, resultando em maior concentração nos seus tecidos. Segundo Nunes et al. (1998), a elevada perda de água durante a armazenagem de morangos tende a mascarar as perdas de ácido ascórbico quando os valores são expressados na base do peso fresco. Ao estudar a pós-colheita de morangos cv. Chandler, Oso Grande e Sweet Charlie, Nunes et al. (1995) observaram que o aparente aumento no conteúdo de ácido ascórbico total, expresso na base do peso fresco durante a armazenagem, foi creditado mais à perda de água dos frutos do que ao aumento real nos teores desse ácido. Entretanto, no presente estudo, os teores de ácido ascórbico diminuíram com o tempo de armazenagem. No experimento 2, diferenças significativas também foram verificadas entre os tempos de armazenagem. Aos 7 dias de armazenagem, o teor de ácido ascórbico encontrado foi de 57,0 mg/100 g, contra $52,5 \mathrm{mg} / 100 \mathrm{~g}$ aos 14 dias. Essa variação não foi detectada pelo teste $t$, o qual não mostrou diferenças significativas nos teores de ácido ascórbico da amostra inicial $(56,5 \mathrm{mg} / 100 \mathrm{~g})$, tanto em relação aos tratamentos armazenados por 7 dias $(57,0 \mathrm{mg} / 100 \mathrm{~g})$ como em relação aos armazenados por 14 dias (52,5 mg/100 g). Cardello \& Cardello (1998) também observaram uma diminuição gradual do conteúdo de vitamina $\mathrm{C}$ com o amadurecimento de mangas. Esse autor cita que a determinação do conteúdo de ácido ascórbico em vegetais é importante, pois, sendo a vitamina mais termolábil, sua presença no alimento indica que provavelmente os demais nutrientes também estão sendo preservados. A inexistência de diferença significativa entre a amostra inicial e os tratamentos armazenados por 14 dias deve ser atri- buída à perda de água, pois, conforme descrito anteriormente, os frutos armazenados por 14 dias apresentaram perda de peso quase três vezes maior do que os armazenados por 7 dias. O resultado disso é uma maior concentração de ácido ascórbico por grama de tecido, resultando em valores semelhantes aos observados no início do experimento.

Em relação aos açúcares totais, no experimento 1, houve diferenças significativas, tanto entre tempos de armazenagem como entre composições da atmosfera (Tabela 2). Os maiores teores de açúcares totais, observados nos tratamentos com ar ambiente, devem ter ocorrido em virtude da elevada perda de água pelos frutos (superior a 17\%), aumentando a concentração de açúcares por grama de tecido. Quanto ao fator tempo de armazenagem, houve aumento significativo dos teores de açúcares totais do início do experimento até os 7 dias $(40,1 \mathrm{mg} / \mathrm{g}$ e $48,6 \mathrm{mg} / \mathrm{g}$, respectivamente), seguido de uma redução significativa aos 14 dias (39,0 mg/g). Os menores valores observados aos 14 dias podem estar relacionados com o maior consumo de carboidratos de reserva dos frutos, para manter a atividade respiratória. No experimento 2 , diferenças significativas foram encontradas apenas entre tempos de armazenagem (Tabela 4). O aparente aumento nos teores de açúcares durante o armazenamento pode não representar o comportamento dos frutos. Isso porque os morangos armazenados por 14 dias apresentaram perda de peso quase três vezes maior em relação aos armazenados por 7 dias, ocorrendo, portanto, a concentra-

Tabela 4. Teores de açúcares totais de morangos cv. Oso Grande mantidos em diferentes composições iniciais de atmosferas e tempos de armazenagem refrigerada a $0^{\circ} \mathrm{C}$ (Experimento 2). Médias de quatro repetições. Porto Alegre, $2001^{(1)}$.

\begin{tabular}{cc}
\hline Tempos de armazenagem (dias) & Açúcares totais $(\mathrm{mg} / \mathrm{g})$ \\
\hline 7 & $35,2 \mathrm{~b}$ \\
14 & $40,2 \mathrm{a}$ \\
$0^{(2)}$ & $32,6 \mathrm{~A}$ \\
7 & $35,2 \mathrm{~A}$ \\
$0^{(2)}$ & $32,6 \mathrm{~B}$ \\
14 & $40,2 \mathrm{~A}$ \\
\hline
\end{tabular}

(1)Médias seguidas de mesma letra minúscula não diferem entre si pelo teste de Duncan a 5\% de probabilidade; médias seguidas de mesma letra maiúscula não diferem entre si pelo teste t a $5 \%$ de probabilidade. ${ }^{(2)}$ Amostra tomada no dia da instalação do experimento (23/01/2001). 
ção de açúcares por grama de amostra. É possível também que esses incrementos aos 14 dias sejam devidos à perda de água, mascarando os resultados quando expressos na base da matéria fresca. Na comparação da média da amostra inicial, pelo teste $\mathrm{t}$, com as médias dos tratamentos armazenados por 7 e 14 dias, os resultados não mostraram aumento significativo dos teores de açúcares totais até os 7 dias, mas revelaram que os tratamentos armazenados por até 14 dias apresentaram valores mais elevados de açúcares totais (Tabela 4). Esperava-se que os teores de açúcares tendessem a diminuir, em decorrência do consumo das reservas de carboidratos. Estes resultados divergem dos obtidos por El-Kazzaz et al. (1983) e Li \& Kader (1989), que não observaram diferenças significativas nos teores de sólidos solúveis em morangos armazenados a $2^{\circ} \mathrm{Ce} 0^{\circ} \mathrm{C}$ por até 14 e 21 dias, respectivamente.

\section{Conclusões}

1. O emprego de atmosferas com concentrações iniciais de $3 \mathrm{kPaO}_{2}+10 \mathrm{kPa} \mathrm{CO}$ ou $5 \mathrm{kPa} \mathrm{O}_{2}+$ $15 \mathrm{kPa} \mathrm{CO}$, associado à refrigeração a $0^{\circ} \mathrm{C}$, permite aumentar o tempo de conservação e manter a qualidade de morangos cv. Oso Grande por até sete dias.

2. A utilização de embalagens de polietileno de $90 \mu \mathrm{m}$ de espessura, como meio para a modificação de atmosferas a partir de concentrações iniciais do ar ambiente, associada ao uso de refrigeração a $0^{\circ} \mathrm{C}$, mantém a qualidade de morangos cv. Oso Grande por até sete dias.

\section{Referências}

BOTREL, N. Sistemas de armazenamento. Informe Agropecuário, Belo Horizonte, v. 17, n. 180, p. 9-13, 1994.

CARDELLO, H. M. A. B.; CARDELLO, L. Teor de vitamina $\mathrm{C}$, atividade de ascorbato oxidase e perfil sensorial de manga (Mangifera indica L.) var. Haden, durante o amadurecimento. Ciência e Tecnologia de Alimentos, Campinas, v. 18, n. 2, p. 211-217, 1998.

DUBOIS, M.; GILLES, K. A.; HAMILTON, J. K.; REBERS, P. A.; SMITH, F. Colorimetric method for determination of sugars and related substances. Analytical Biochemistry, Orlando, v. 28, p. 350-356, 1956.

EL-KAZZAZ, M. K.; SOMMER, N. F.; FORTLAGE, R. J. Effect of different atmospheres on postharvest decay and quality of fresh strawberries. Phytopathology, Saint Paul, v. 73, n. 2, p. 282-285, 1983.

GARCÍA, J. M.; MEDINA, R. J.; OLÍAS, J. M. Quality of strawberries automatically packed in different plastic films. Journal of Food Science, Chicago, v. 63, n. 6, p. 1037-1041, 1998.

HAMSON, A. R. Measuring firmness of tomatoes in a breeding program. Proceeding of the American Society for Horticultural Science, Alexandria, v. 60, p. 425-433, 1952.

KADER, A. A. Postharvest technology of horticultural crops. 2. ed. Oakland: University of California, 1992. $296 \mathrm{p}$.

KE, D.; EL-SHEIKN, T.; MATEOS, M.; KADER, A. A. Anaerobic metabolism of strawberries under elevated $\mathrm{CO}_{2}$ and reduced $\mathrm{O}_{2}$ atmospheres. Acta Horticulturae, Wageningen, v. 343, p. 93-99, 1993.

KIM, G. H.; WILLS, R. B. Interaction of enhanced carbon dioxide and reduced ethylene on the storage life of strawberries. Journal of Horticultural Science and Biotechnology, Ashford, v. 73, n. 2, p. 181-184, 1998.

LARSEN, M.; WATKINS, C. B. Firmness and aroma composition of strawberries following short-term height carbon dioxide treatments. HortScience, Alexandria, v. 30, n. 2, p. 303-305, 1995.

LI, C.; KADER, A. A. Residual effects of controlled atmospheres on postharvest physiology and quality of strawberries. Journal of the American Society for Horticultural Science, Alexandria, v. 114, n. 4, p. 629634, 1989.

NUNES, M. C. N.; BRECHT, J. K.; MORAIS, A. M. M. B.; SARGENT, S. A. Controlling temperature and water loss to maintain ascorbic acid levels in strawberries during postharvest handling. Journal of Food Science, Chicago, v. 63, n. 6, p. 1033-1036, 1998.

NUNES, M. C. N.; BRECHT, J. K.; MORAIS, A. M. M. B.; SARGENT, S. A. Physical and chemical quality characteristics of strawberries after storage are reduced by a short delay to cooling. Postharvest Biology and Technology, Amsterdam, v. 6, p. 17-28, 1995. 
NUNES, M. C. N.; MORAIS, A. M. M. B. Quality of strawberries after storage in controlled atmospheres at above optimum storage temperatures. Proceeding of the Florida State Society for Horticultural Science, Florida, v. 108, p. 273-277, 1995.

SANZ, C.; PÉREZ, A. G.; OLÍAS, R.; OLÍAS, J. M. Quality of strawberries packed with perforated polypropylene. Journal of Food Science, Chicago, v. 64, n. 4, p. 748-752, 1999.

SMITH, R. B. Controlled atmosphere storage of "Readcoat" strawberry fruit. Journal of the American
Society for Horticultural Science, Alexandria, v. 117, n. 2, p. 260-264, 1992.

SMITH, R. B.; SKOG, L. J. Postharvest carbon dioxide treatment enhances firmness of several cultivars of strawberry. HortScience, Alexandria, v. 27, n. 5, p. 420421, 1992.

TEREDA, M.; WATANABE, Y.; KUNITOMA, M.; HAYASHI, E. Differential rapid analysis of ascorbic acid and ascorbic acid 2-sulfate by dinitrophenylhydrazine method. Analytical Biochemistry, Orlando, v. 84, p. 604608, 1978. 